\title{
Microlocal limits of Eisenstein functions away from the unitarity axis
}

\author{
Semyon Dyatlov
}

\begin{abstract}
We consider a surface $M$ with constant curvature cusp ends and its Eisenstein functions $E_{j}(\lambda)$. These are the plane waves associated to the $j$ th cusp and the spectral parameter $\lambda,\left(\Delta-1 / 4-\lambda^{2}\right) E_{j}=0$. We prove that as $\operatorname{Re} \lambda \rightarrow \infty$ and $\operatorname{Im} \lambda \rightarrow v>0, E_{j}$ converges microlocally to a certain naturally defined measure decaying exponentially along the geodesic flow. In particular, for a surface with one cusp and a sequence of $\lambda$ 's corresponding to scattering resonances, we find the microlocal limit of resonant states with energies away from the real line. This statement is similar to quantum unique ergodicity (QUE), which holds in certain other situations; however, the proof uses only the structure of the infinite ends, not the global properties of the geodesic flow. As an application, we also show that the scattering matrix tends to zero in strips separated from the real line.
\end{abstract}

Mathematics Subject Classification (2010). Primary 35P20, 35P25; Secondary 30F35.

Keywords. Surfaces with cusps, Eisenstein functions, semiclassical measures.

\section{Introduction}

Concentration of eigenfunctions of the Laplacian in phase space dates back to the papers of Schnirelman [25], Colin de Verdière [3], and Zelditch [30]. Their quantum ergodicity $(\mathrm{QE})$ result states that on a closed Riemannian manifold whose geodesic flow is ergodic with respect to the Liouville measure, a density one subsequence of eigenfunctions converges microlocally to this measure. For manifolds with boundary, QE was proved in a special case by Gérard-Leichtnam [7] and in general by ZelditchZworski [33]. The paper [7] used the semiclassical defect measure approach taken here.

The papers [13], [16], [17], [26], and [31] studied the question for finite area hyperbolic surfaces, that is hyperbolic quotients with cusps. In particular, [31] established QE for any such surface, if embedded eigenfunctions are augmented with Eisenstein functions on the real line; the latter parametrize continuous spectrum of the Laplacian arising from the presence of cusps. For the modular surface one has a stronger statement of quantum unique ergodicity (QUE): any sequence of Hecke- 
Maass forms (see[16] and [26]) or Eisenstein functions on the real line (see [17] and [13]) converges microlocally to the Liouville measure. Guillarmou and Naud [9] have recently studied equidistribution of Eisenstein functions for convex co-compact hyperbolic manifolds; that is, in the presence of funnels, but not cusps. Finally, after this paper had been posted as a preprint on arXiv, an interesting preprint [22] appeared that addresses similar questions to those we study, in particular proving our Theorem 1, in the special case of the modular surface. See [19], [24], and [32] for recent reviews of other results.

The present paper considers an arbitrary surface with cusps and studies phase space concentration of Eisenstein functions for the spectral parameter $\lambda$ in the upper half-plane, away from the real line. We show that for a given cusp and a given limit $v>0$ of $\operatorname{Im} \lambda$, there is only one limiting measure - see Theorem 1. This statement is similar to that of QUE; however, in contrast with the Q(U)E facts listed above, we do not use any global properties of the geodesic flow, such as hyperbolicity or ergodicity. Instead, we represent Eisenstein functions as plane waves; that is, the sum of 'incoming' and 'outgoing' waves, where the 'incoming wave' depends only at the structure of the manifold at infinity. The main idea of the paper can be summarized as follows: the microlocal limit of a plane wave is obtained by taking the natural measure corresponding to the 'incoming' part of this wave and propagating it along the geodesic flow. The key difference from the case $\operatorname{Im} \lambda \rightarrow 0$ is that the corresponding semiclassical measures are exponentially decaying, rather than invariant, along the geodesic flow.

We restrict ourselves to the case of surfaces with exact cusp ends. However, the method of the proof could potentially be applied to complete Riemannian manifolds with a variety of infinite ends, or even to more general self-adjoint semiclassical differential operators, as long as a notion of plane waves exists. For example, in the case of a compactly supported metric perturbation of the Euclidean metric on $\mathbb{R}^{3}$, plane waves are solutions to the equation

$$
\left(\Delta_{x}-\lambda^{2}\right) E(\lambda, \omega ; x)=0, \quad \omega \in \mathbb{S}^{2}, \lambda \in \mathbb{C},
$$

that have the following form near infinity:

$$
E(\lambda, \omega ; x)=e^{-i \lambda \omega \cdot x}+E^{\prime \prime}(\lambda, \omega ; x),
$$

where $E^{\prime \prime}$ is outgoing (for $\operatorname{Im} \lambda>0$, this means that it lies in $L^{2}$ of the whole space). The limiting measure for $E(\lambda, \omega ; x)$ with $\operatorname{Re} \lambda \rightarrow+\infty, \operatorname{Im} \lambda \rightarrow v>0$, and $\omega \rightarrow \omega_{0} \in \mathbb{S}^{2}$ can be obtained by propagating forward along the flow the measure $e^{2 v \omega_{0} \cdot x} d x$ defined on $\left\{|x| \gg 1, \xi=-\omega_{0}\right\}$, similarly to the definition of the measure $\mu_{j v}$ in (1.11) below.

Our motivation comes from the natural question of quantum ergodicity of resonant states. These replace eigenfunctions on non-compact manifolds, and their equidistribution in phase space was studied in the model of quantized open maps by Nonnenmacher-Rubin [20]. In a similar setting, Demers and Young [5] have observed that a conditionally invariant measure for a billiard with a hole is determined 
entirely by its behavior in the hole; this is somewhat parallel to the main idea of our paper presented above. See also an interesting physics paper by Keating et al. [14].

As stated in Theorem 3, microlocal convergence for Eisenstein functions away from the real line yields a microlocal convergence result for resonant states with complex energies at a fixed distance from the real line. Although this does not address the $\operatorname{Im} \lambda \rightarrow 0$ case, satisfied by most resonances (see Conjecture 1 below), it seems to be the first result on microlocal convergence of resonant states of differential operators.

We proceed to a rigorous formulation of the results. Let $(M, g)$ be a twodimensional complete Riemannian manifold with cusp ends; that is, $M$ is the union of a compact set and finitely many cusp regions $\ell_{1}, \ldots, \ell_{m}$, where each $\ell_{j}$ possesses a system of canonical coordinates

$$
(r, \theta) \in(R, \infty) \times \mathbb{S}^{1}, \quad \mathbb{S}^{1}=\mathbb{R} /(2 \pi \mathbb{Z}),
$$

with $R$ some constant, such that the metric $g$ on $\ell_{j}$ has the form

$$
g=d r^{2}+e^{-2 r} d \theta^{2} .
$$

A classical example of such $M$ is a finite area hyperbolic surface without conic points. In fact, the present paper applies to finite area hyperbolic quotients $\Gamma \backslash \mathbb{H}$ with conic points as well, as one can get rid of these by passing to a finite covering space (the cone angles are rational multiples of $\pi$, as the corresponding elliptic transformation has to generate a discrete subgroup of $\operatorname{PSL}(2, \mathbb{R})$ - see for example [1], Chapter 2).

Let $\Delta$ be the (nonnegative) Laplace-Beltrami operator corresponding to the metric $g$; this operator is self-adjoint, its spectrum is contained in $[0, \infty)$, and the spectrum in $[0,1 / 4)$ consists of finitely many eigenvalues; see [18], Section 1 . The Eisenstein functions

$$
E_{j}(\lambda), \quad j=1, \ldots, m, \operatorname{Im} \lambda>0, \lambda \notin(0, i / 2],
$$

are unique solutions to the equation ${ }^{1}$

$$
\left(\Delta-1 / 4-\lambda^{2}\right) u=0, \quad u \in C^{\infty}(M),
$$

that satisfy

$$
u-1 e_{j} e^{(1 / 2-i \lambda) r} \in L^{2}(M) .
$$

Here $1 e_{j}$ is the indicator function of the cusp region $\ell_{j}$. To define $L^{2}(M)$, we use the volume form Vol induced by $g$. See Section 3 for details.

We would like to study in particular the (weak) limit of the measure $\left|E_{j}(\lambda)\right|^{2} d$ Vol as $\lambda$ tends to the infinity in a certain way. It turns out that it is more natural to study

\footnotetext{
${ }^{1}$ For hyperbolic quotients, is more conventional to use the parameter $s=1 / 2-i \lambda$, with $\lambda^{2}+1 / 4=$ $s(1-s)$ and the physical region $\{\operatorname{Im} \lambda>0\}$ corresponding to $\{\operatorname{Re} s>1 / 2\}$. We use the parameter $\lambda$ to emphasize that our argument belongs to general scattering theory and is applicable to other cases such as the Euclidean case mentioned above.
} 
microlocal convergence of $E_{j}(\lambda)$ in the sense of semiclassical defect measures. A definition of these for a compact manifold can be found in [34], Chapter 5; we use semiclassical notation presented in Section 2. Since $M$ is noncompact and $E_{j}$ does not lie in $L^{2}$, we have to insert compactly supported cutoffs into the following definition.

Definition 1. Let $h_{n}$ be a sequence of positive numbers tending to zero and $u_{n}$ be a sequence of functions on $M$ bounded uniformly on $L^{2}(K)$ for each compact $K \subset M$. (The Eisenstein functions satisfy this property by (3.8).) We say that the sequence $u_{n}$ converges microlocally to some Radon measure $\mu$ on $T^{*} M$ if, for each pseudodifferential operator $A(h) \in \Psi^{0}$ with principal symbol $a \in C^{\infty}\left(T^{*} M\right)$, and each $\chi \in C_{0}^{\infty}(M ; \mathbb{R})$, we have

$$
\left\langle A\left(h_{n}\right) \chi u_{n}, \chi u_{n}\right\rangle_{L^{2}(M)} \longrightarrow \int_{T^{*} M} \chi^{2} a d \mu .
$$

The measure $\mu$ is called the semiclassical measure associated to the sequence $u_{n}$.

In particular, we can take as $A(h)$ the multiplication operator by $a(z) \in C_{0}^{\infty}(M)$ :

$$
\int a(z)\left|u_{n}\right|^{2} d \mathrm{Vol} \longrightarrow \int_{T^{*} M} a(z) d \mu .
$$

In other words, the measure $\left|u_{n}\right|^{2} d$ Vol converges weakly to the pushforward of $\mu$ under the projection $\pi: T^{*} M \rightarrow M$.

We list several basic properties of semiclassical measures; we do not use them in the present paper, but mention them to explain why the measure $\mu_{j v}$ defined below is a reasonable candidate for the microlocal limit of Eisenstein functions. Assume that $\lambda(h)$ is a family of complex numbers satisfying ${ }^{2}$

$$
\operatorname{Re} \lambda(h)=h^{-1}, \quad \operatorname{Im} \lambda(h) \longrightarrow v>0 \quad \text { as } h \rightarrow 0 .
$$

Note that by (1.2),

$$
\left(h^{2} \Delta-h^{2} / 4-(1+i h \operatorname{Im} \lambda(h))^{2}\right) E_{j}(\lambda(h))=0 .
$$

However, $P(h)=h^{2} \Delta$ is a semiclassical differential operator of order 2; its principal symbol, which we denote by $p$, is the square of the norm induced by the metric $g$ on the cotangent bundle. Therefore, the set $\{p=1\}$ is the cosphere bundle $S^{*} M$, consisting of covectors of length 1 ; moreover, if $\exp (t V)$ is the geodesic flow on $T^{*} M$ and $\exp \left(t H_{p}\right)$ is the Hamiltonian flow of $p$, then

$$
\exp \left(t H_{p}\right)=\exp (2 t V) \quad \text { on } S^{*} M .
$$

\footnotetext{
${ }^{2}$ Same methods apply with $\operatorname{Re} \lambda \rightarrow-\infty$, with signs in certain formulas inverted. The corresponding semiclassical measures are exponentially increasing along the geodesic flow and concentrated on the outgoing, rather than incoming, set $\mathcal{A}_{j}^{+}$.
} 
Let $h_{n}$ be any sequence tending to zero such that the sequence $E_{j}\left(\lambda\left(h_{n}\right)\right)$ converges microlocally to some Radon measure $\mu$. Applying the methods of proof of [34], Section 5.2, to (1.5), one can get the following properties:

(i) $\mu$ is supported on the cosphere bundle: $\mu\left(T^{*} M \backslash S^{*} M\right)=0$;

(ii) $\mu$ decays exponentially along the geodesic flow: for each $A \subset T^{*} M$,

$$
\mu(\exp (t V) A)=e^{-2 v t} \mu(A) .
$$

We have exponential decay, rather than invariance under the flow, in (1.7), because the imaginary part of the operator in (1.5) is asymptotic to $-2 h v$. Note that there exist multiple measures satisfying properties (i) and (ii) above; each geodesic emanating directly from some cusp carries such a measure. In fact, it can be proved ${ }^{3}$ that every Radon measure $\mu$ satisfying properties (i) and (ii) is supported on the union $\mathcal{A}^{-}$of the incoming sets $\mathcal{A}_{j}^{-}$defined below and thus it can be written as an integral, over some measure on the circle, of measures supported on geodesics emanating directly from the cusps. The main result of the paper is that there is only one possible semiclassical measure for Eisenstein functions for fixed $j$ and $v>0$.

Theorem 1. Let $h>0$ be a small parameter tending to zero, and assume that $\lambda(h)$ satisfies (1.4). Then for each $A(h) \in \Psi^{0}$ with semiclassical principal symbol $a$, and each $\chi \in C_{0}^{\infty}(M ; \mathbb{R})$, we have as $h \rightarrow 0$,

$$
\left\langle A(h) \chi E_{j}(\lambda(h)), \chi E_{j}(\lambda(h))\right\rangle_{L^{2}(M)} \longrightarrow \int \chi^{2} a d \mu_{j v}
$$

and

$$
\left\langle A(h) \chi E_{j}(\lambda(h)), \chi E_{j^{\prime}}(\lambda(h))\right\rangle_{L^{2}(M)} \longrightarrow 0, j \neq j^{\prime} .
$$

Here $\mu_{j v}$ is the measure defined in (1.11) below.

Together, (1.8) and (1.9) can be interpreted as follows: for any $\alpha \in \mathbb{C}^{m}$, the linear combination $\sum_{j} \alpha_{j} E_{j}(\lambda(h))$ converges microlocally to the measure $\sum_{j}\left|\alpha_{j}\right|^{2} \mu_{j v}$.

To construct the measure $\mu_{j v}$, we first define the incoming set $\mathcal{A}_{j}^{-}$and the outgoing set $\mathcal{A}_{j}^{+}$. Let $(r, \theta)$ be the canonical coordinates in the cusp $\bigodot_{j}$ and $\left(r, \theta, p_{r}, p_{\theta}\right)$ be the induced system of coordinates on $T^{*} \ell_{j}$; define

$$
\begin{gathered}
\mathcal{A}_{j}^{ \pm}=\left\{\rho \in S^{*} M \mid \exists t>0: G^{ \pm t} \rho \in \widehat{\mathcal{A}}_{j}^{ \pm}\right\}, \\
\widehat{\mathcal{A}}_{j}^{ \pm}=\left\{\left(r, \theta, p_{r}, p_{\theta}\right) \in T^{*} \mathcal{C}_{j} \mid p_{r}= \pm 1, p_{\theta}=0\right\} .
\end{gathered}
$$

\footnotetext{
${ }^{3}$ Here is a sketch of the proof. Let $K=\{r \leq R+1\} \subset S^{*} M$; since $K$ is compact, we have $\mu(K)=$ $c<\infty$. Then by (1.7) for each $l \in \mathbb{N}, \mu(\exp (l V) K)=e^{-2 v l} c$ and thus the series $\sum_{l} \mu(\exp (l V) K)$ converges. By Borel-Cantelli lemma, we get the required statement $\mu\left(S^{*} M \backslash \mathcal{A}^{-}\right)=0$ if we show that, for each $\rho \in S^{*} M \backslash \mathcal{A}^{-}$, there exist infinitely many $l \in \mathbb{N}$ such that $\exp (-l V) \rho \in K$. The latter follows from the fact that every unit speed backwards geodesic that leaves $K$ enters some cusp region $\mathcal{C}_{j}$; unless this geodesic lies in $\mathcal{A}^{-}$, it will eventually bounce back and return to $K$, where it will spend an interval of length at least 1 , containing the point $\exp (-l V) \rho$ for some integer $l$.
} 
In other words, $\mathcal{A}_{j}^{+}$is the union of all geodesics going directly into the $j$ th cusp and $\mathcal{A}_{j}^{-}$is the union of all geodesics emanating directly from it. Note that $\mathcal{A}_{j}^{ \pm}$need not be closed; in fact, for hyperbolic surfaces each of them is dense in $S^{*} M$. The measure $\mu_{j v}$ is supported on $\mathcal{A}_{j}^{-}$and is constructed as follows: we start with the cylindrical measure $e^{2 v r} d r d \theta$ on $\hat{\mathcal{A}}_{j}^{-}$and propagate it to a measure on the whole $\mathcal{A}_{j}^{-}$using property (ii) of semiclassical measures; the result converges because $v>0$. More formally, for each continuous compactly supported function $a$ on $T^{*} M$, we put

$$
\int_{T^{*} M} a d \mu_{j v}=\lim _{t \rightarrow+\infty} e^{-2 v t} \int_{e_{j}} e^{2 v r}(a \circ \exp (t V))(r, \theta,-1,0) d r d \theta .
$$

It can be seen directly that (1.11) defines a Radon measure satisfying properties (i) and (ii) of semiclassical measures.

The proof of Theorem 1 is based on the representation of Eisenstein functions as plane waves:

$$
E_{j}(\lambda)=E_{j}^{\prime}(\lambda)+E_{j}^{\prime \prime}(\lambda),
$$

where $E_{j}^{\prime}(\lambda)=1 e_{j} \tilde{\chi}(r-R) e^{(1 / 2-i \lambda) r}$ is the 'incoming' and $E_{j}^{\prime \prime}(\lambda)$ the 'outgoing' part; since $\lambda$ is in the upper half-plane, $E_{j}^{\prime \prime}$ is bounded in $L^{2}(M)$ uniformly in $h$. We now consider the semiclassical Schrödinger propagator $e^{i t h \Delta}=e^{i t\left(h^{2} \Delta\right) / h}$; by (1.2), we have formally

$$
e^{i t h \Delta} E_{j}(\lambda)=e^{i t h\left(1 / 4+\lambda^{2}\right)} E_{j}(\lambda) .
$$

Note that $\left|e^{i t h\left(1 / 4+\lambda^{2}\right)}\right|=e^{-2 t \operatorname{Im} \lambda}$ is exponentially decaying as $t \rightarrow+\infty$. For a compactly supported (that is, having compactly supported Schwartz kernel) $A(h) \in$ $\Psi^{0}$,

$$
\left\langle A(h) E_{j}(\lambda), E_{j}(\lambda)\right\rangle=e^{-4 t \operatorname{Im} \lambda}\left\langle e^{i t h \Delta} A(h) e^{-i t h \Delta} E_{j}(\lambda), E_{j}(\lambda)\right\rangle .
$$

However, as $e^{i t h \Delta}$ is unitary and $E_{j}^{\prime \prime}$ is bounded in $L^{2}(M)$, we can replace $E_{j}$ by $E_{j}^{\prime}$ with a remainder exponentially decaying in $t$ :

$$
\left\langle A(h) E_{j}(\lambda), E_{j}(\lambda)\right\rangle=e^{-4 t \operatorname{Im} \lambda}\left\langle e^{i t h \Delta} A(h) e^{-i t h \Delta} E_{j}^{\prime}(\lambda), E_{j}^{\prime}(\lambda)\right\rangle+\mathcal{O}\left(e^{-2 t \operatorname{Im} \lambda}\right) .
$$

(The remainder is $\mathcal{O}\left(e^{-2 t \operatorname{Im} \lambda}\right)$ instead of $\mathcal{O}\left(e^{-4 t \operatorname{Im} \lambda}\right)$, as the intersection of the wavefront set of $e^{i t h \Delta} A(h) e^{-i t h \Delta}$ with $S^{*} M$ lies in $\{r \leq 2 t+T\}$ for some constant $T$ depending on the support of $A(h)$ and thus $e^{i t h \Delta} A(h) e^{-i t h \Delta} E_{j}^{\prime}(\lambda)$ should be of size $e^{2 t \operatorname{Im} \lambda}$.) We can now perform an explicit computation using Egorov's theorem and the formula for $E_{j}^{\prime}$ to see that, as $h \rightarrow 0$, the first term on the right-hand side of (1.13) converges formally to

$$
e^{-4 v t} \int_{\mathscr{C}_{j}} e^{2 v r} \tilde{\chi}(r-R)^{2}(a \circ \exp (2 t V))(r, \theta,-1,0) d r d \theta .
$$

It remains to let $t \rightarrow+\infty$ to obtain (1.8); (1.9) follows by a similar argument. 
There is however a serious gap in the 'proof' presented above; namely, the operator $e^{i t h \Delta}$ is only defined on $L^{2}$ and is not properly supported. Since the function $E_{j}(\lambda)$ does not lie in $L^{2}$, the equation (1.12) does not make any sense. Similarly, the operator $e^{i t h \Delta} A(h) e^{-i t h \Delta}$ is not compactly supported and thus one cannot apply it to $E_{j}^{\prime}(\lambda)$. To fix this gap, we use cutoffs depending on $t$ and on the support of $A(h)$; see Proposition 5.

One could also try to fix the gap discussed in the previous paragraph by using the propagator

$$
U(t)=\left(\begin{array}{cc}
\cos (t \sqrt{\Delta}) & \sin (t \sqrt{\Delta}) / \sqrt{\Delta} \\
-\sqrt{\Delta} \sin (t \sqrt{\Delta}) & \cos (t \sqrt{\Delta})
\end{array}\right)
$$

for the Cauchy problem for the wave equation

$$
\left(D_{t}^{2}-\Delta_{x}\right) u(t, x)=0, \quad t \in \mathbb{R}, x \in M .
$$

Indeed, since (1.14) has finite speed of propagation, the elements of the matrix $U(t)$ act $C^{\infty}(M) \rightarrow C^{\infty}(M)$. Define $\sqrt{\lambda^{2}+1 / 4}$ to have positive real part, so that it is equal to $\lambda+\mathcal{O}(h)$. By (1.2), the function $e^{i t \sqrt{\lambda^{2}+1 / 4}} E_{j}(\lambda)$ solves (1.14) and for

$$
\vec{E}_{j}(\lambda)=\left(1, i \sqrt{\lambda^{2}+1 / 4}\right) E_{j}(\lambda)
$$

we have $U(t) \vec{E}_{j}(\lambda)=e^{i t \sqrt{\lambda^{2}+1 / 4}} \vec{E}_{j}(\lambda)$. One could then try to argue as above, using that $U(t)$ is unitary on $\dot{H}^{1}(M) \oplus L^{2}(M)$ to estimate the contribution of $E_{j}^{\prime \prime}(\lambda)$, and using that $U(-t)\left(1, i \sqrt{\lambda^{2}+1 / 4}\right) E_{j}^{\prime}(\lambda)$ is a Lagrangian state associated to propagating $\hat{\mathcal{A}}_{j}^{-}$by $\exp (t V)$, to calculate the contribution of $E_{j}^{\prime}(\lambda)$.

As an application of Theorem 1, we derive a bound on the scattering matrix $S(\lambda)$. For each two cusps $\ell_{j}, \ell_{j^{\prime}}$, define $S_{j j^{\prime}}(\lambda)$ by

$$
E_{j} \mid \ell_{j^{\prime}}(\lambda ; r, \theta)=\delta_{j j^{\prime}} e^{(1 / 2-i \lambda) r}+S_{j j^{\prime}}(\lambda) e^{(1 / 2+i \lambda) r}+\cdots,
$$

where $(r, \theta)$ are canonical coordinates on $\ell_{j^{\prime}}, \delta$ is the Kronecker delta, and the dots denote the terms corresponding to terms with $k \neq 0$ in the Fourier series expansion (3.1) of $E_{j} \mid e_{j^{\prime}}$ in the $\theta$ variable.

Theorem 2. Consider two cusps $\ell_{j}, \ell_{j^{\prime}}$ and assume that $\mu_{j v}\left(\mathcal{A}_{j^{\prime}}^{+}\right)=\emptyset$ (in particular, this is true for hyperbolic surfaces, as $\mathcal{A}_{j^{\prime}}^{+} \cap \mathcal{A}_{j}^{-}$consists of countably many geodesics). Then for $\lambda(h)$ satisfying (1.4),

$$
S_{j j^{\prime}}(\lambda(h)) \longrightarrow 0 \quad \text { as } h \rightarrow 0 .
$$

In other words,

$$
S_{j j^{\prime}}(\lambda)=o(1), \quad 0<C^{-1}<\operatorname{Im} \lambda<C, \operatorname{Re} \lambda \rightarrow \infty .
$$


This estimate is not always optimal: in the special case of the modular surface $M=$ $\operatorname{PSL}(2, \mathbb{Z}) \backslash \mathbb{Q}$, the scattering coefficient $S(\lambda)$ is related to the Riemann zeta function by the formula [27], Section 2.18,

$$
S(\lambda)=\sqrt{\pi} \frac{\zeta(-2 i \lambda) \Gamma(-i \lambda)}{\zeta(1-2 i \lambda) \Gamma(1 / 2-i \lambda)} .
$$

Given that both $\zeta(z)$ and $\zeta^{-1}(z)$ are bounded in every half-plane $\left\{\operatorname{Re} z>1+C^{-1}\right\}$ (either by Dirichlet series or by Euler product representation), the basic bound on the zeta function in the critical strip (see [27], eq. (5.1.4)) gives

$$
|S(\lambda)|=\mathcal{O}\left(|\lambda|^{-\min (\operatorname{Im} \lambda, 1 / 2)-}\right), \quad \operatorname{Im} \lambda \geq C^{-1} .
$$

The bound (1.15) is optimal for $\operatorname{Im} \lambda>1 / 2$, and no optimal bounds are known for $0<\operatorname{Im} \lambda<1 / 2$. It would be interesting to see if semiclassical methods can yield an effective bound on the scattering coefficients, and compare such bound to (1.15).

Finally, we address the question of microlocal convergence of resonant states. Assume that for some $\lambda$, the matrix $S(\lambda)$ is not invertible; that is, there exists $\alpha \in$ $\mathbb{C}^{m} \backslash\{0\}$ such that, for each $j^{\prime}$,

$$
\sum_{j} \alpha_{j} S_{j j^{\prime}}(\lambda)=0
$$

This is equivalent to saying that $-\lambda$ is a resonance; i.e., a pole of the meromorphic continuation of the resolvent $\left(\Delta-1 / 4-\lambda^{2}\right)^{-1}$ to the lower half-plane (see for example [18], Section 5). Moreover, a resonant state at $-\lambda$ is given by $\sum_{j} \alpha_{j} E_{j}(\lambda)$. Theorem 1 immediately implies the following result.

Theorem 3. Assume that $-\lambda_{n}$ is a sequence of resonances satisfying (1.4) for some $h_{n} \rightarrow 0$. Let $u_{n}$ be a sequence of corresponding resonant states and assume that it converges microlocally to some measure $\mu$. Then $\mu$ is a linear combination of the measures $\mu_{1 v}, \ldots, \mu_{m v}$ defined by (1.11).

The fact that semiclassical measures for resonant states are exponentially decaying along the geodesic flow is parallel to Theorem 4 of [21]; a similar fact has been obtained in the setting of quantized open maps in [20]. However, the concentration statement (see[21], eq. (1.15)) is vacuous in our case, as the set $\Gamma_{E}^{-}$from [21] (not $\Gamma_{E}^{+}$, as $\left.\operatorname{Re}(-\lambda)<0\right)$ is the whole cosphere bundle. In fact, [21] heavily use the fact that resonant states are outgoing, while Eisenstein functions studied in the present paper need not satisfy the outgoing condition (which in our case is (1.16)).

For surfaces with only one cusp, resonant states away from the real line have to converge microlocally to a single measure; however, we do not address the question of the behavior of the kernel of $S(\lambda)$ for multiple cusps. For other types of infinite ends (such as the convex cocompact case), the scattering matrix $S(\lambda)$ is replaced by an operator acting on a certain infinite dimensional Hilbert space. The distribution of 
resonances in strips of fixed size and the behavior of the kernel of $S(\lambda)$ is controlled by the trapping phenomenon in the compact part of our manifold, while the restriction on the set of possible semiclassical measures provided in this paper only uses behavior at infinity.

Finally, it would be natural to ask an analogue of the quantum ergodicity question: where do most resonant states microlocally converge if we drop the restriction $\operatorname{Im} \lambda \rightarrow v>0$ ? Theorem 3 does not provide the answer because most resonances are located $o(1)$ close to the real line. To make this last statement precise, we assume that there are only finitely many embedded eigenvalues (which is true under certain genericity assumptions - see [2], Théorème 7, and [23]), let Res be the set of resonances, counted with multiplicities, and recall the Weyl law; see [18], eq. (0.5):

$$
\left|\operatorname{Res} \cap\left\{|\lambda| \leq h^{-1}\right\}\right|=\frac{\operatorname{Area}(M)}{2 \pi} h^{-2}(1+o(1)) .
$$

We claim that for each $\varepsilon>0$,

$$
\left|\operatorname{Res} \cap\left\{|\lambda| \leq h^{-1}, \operatorname{Im} \lambda<-\varepsilon\right\}\right|=o\left(h^{-2}\right) .
$$

The proof of (1.17) is based on Corollary 3.29 of [18]:

$$
\sum_{\lambda \in \operatorname{Res}} \frac{|\operatorname{Im} \lambda|}{|\lambda|^{2}}<\infty
$$

If (1.17) is false, then there exist $\varepsilon, \delta>0$ and a sequence $h_{j} \rightarrow 0$ such that

$$
\left|\operatorname{Res} \cap\left\{|\lambda| \leq h_{j}^{-1}, \operatorname{Im} \lambda<-\varepsilon\right\}\right| \geq 2 \delta h_{j}^{-2} \text {. }
$$

However, by the upper bound provided by the Weyl law, there exists a constant $C_{0}$ such that

$$
\left|\operatorname{Res} \cap\left\{C_{0}^{-1} h_{j}^{-1} \leq|\lambda| \leq h_{j}^{-1}, \operatorname{Im} \lambda<-\varepsilon\right\}\right| \geq \delta h_{j}^{-2} .
$$

We pass to a subsequence of $h_{j}$ such that $h_{j} / h_{j+1}>C_{0}$. Then the sets of resonances counted in (1.19) for different $j$ do not intersect each other, and the sum of $|\operatorname{Im} \lambda| /|\lambda|^{2}$ over each of these sets is bounded from below by $\varepsilon \delta$, contradicting (1.18).

By (1.17), a density one subsequence of resonances converges to the real line; the corresponding semiclassical measures are invariant with respect to the geodesic flow and a natural candidate is the Liouville measure.

Conjecture 1. Assume that $M$ is a surface with cusp ends whose geodesic flow is ergodic with respect to the Liouville measure $\mu_{0}$. Then there exists a density one subsequence of resonant states in any strip $\{\operatorname{Im} \lambda>-C\}$ converging microlocally to $\mu_{0}$.

The proof of (1.17) and Conjecture 1 have been suggested by Maciej Zworski. 
The paper is organized as follows. In Section 2, we review some notation and facts from semiclassical analysis. In Section 3, we present basic facts about Eisenstein functions and prove Theorems 1 and 2. Finally, in Section 4, we consider the special case of finite area hyperbolic surfaces and describe the canonical measures $\mu_{j v}$ from (1.11) via the action of the fundamental group of $M$; we also prove Theorem 1 in this case for $\operatorname{Im} \lambda>1 / 2$ using the classical definition of Eisenstein functions as series.

Acknowledgements. I would like to thank Maciej Zworski for his interest in the project and helpful advice. I would also like to thank Steven Zelditch for several helpful discussions on the nature of measures satisfying (1.7) and in particular directing me to the formula (4.5), and Stéphane Nonnenmacher and Yves Colin de Verdière for many suggestions for improving the manuscript. I am especially thankful to an anonymous referee for reading the paper carefully and many useful remarks. Finally, I am grateful for partial support from NSF grant DMS-0654436.

\section{Semiclassical preliminaries}

In this section, we briefly review the portions of semiclassical analysis used below; the reader is referred to [34] for a detailed account on the subject.

We assume that $h>0$ is a parameter, the smallness of which is implied in all statements below. Consider the algebra $\Psi^{s}\left(\mathbb{R}^{d}\right)$ of pseudodifferential operators with symbols in the class $S^{s}\left(\mathbb{R}^{d}\right)$, defined as follows: a function $a(x, \xi ; h)$ smooth in $(x, \xi) \in \mathbb{R}^{2 d}$ lies in this class if and only if for each compact set $K \subset \mathbb{R}^{d}$ and each multiindices $\alpha, \beta$, there exists a constant $C_{\alpha \beta K}$ such that

$$
\sup _{(x, \xi) \in K \times \mathbb{R}^{d}}\left|\partial_{\alpha}^{x} \partial_{\beta}^{\xi} a(x, \xi ; h)\right| \leq C_{\alpha \beta K}\langle\xi\rangle^{s-|\beta|} .
$$

The only difference with the invariant symbol classes studied in [34], Section 9.3, is that we do not require uniform bounds as $x \rightarrow \infty$. However, this does not matter in our situation, as we will mostly use compactly supported operators; e.g. those operators whose Schwartz kernels are compactly supported in $\mathbb{R}^{d} \times \mathbb{R}^{d}$. As in [34], Section 13.2, we can define the algebra $\Psi^{s}(M)$ for any manifold $M$. The compactly supported elements of $\Psi^{s}(M)$ act $H_{\hbar, \text { loc }}^{t}(M) \rightarrow H_{\hbar \text {,comp }}^{t-s}(M)$ with norm $\mathcal{O}(1)$ as $h \rightarrow 0$, where $H_{\hbar, \text { loc }}^{t}$ and $H_{\hbar \text {,comp }}^{t-s}$ are semiclassical Sobolev spaces.

To avoid discussion of simultaneous behavior of symbols as $\xi \rightarrow \infty$ and $h \rightarrow 0$, we further require that the symbols of elements of $\Psi^{s}$ are classical, in the sense that they posses an asymptotic expansion in powers of $h$, with the term next to $h^{k}$ lying in $S^{s-k}$ (see [6], Section 2.1, for details). Following [28], Section 2, we introduce the fiber-radial compactification $\bar{T}^{*} M$ of the cotangent bundle. (The use of $\bar{T}^{*} M$ slightly simplifies the statement of Proposition 1 below. However, as all other microlocal 
analysis is happening in a compact subset of $T^{*} M$, one could easily avoid fiber-radial compactification if needed.) Each $A \in \Psi^{s}$ has an invariantly defined (semiclassical) principal symbol $\sigma(A)=a \in C^{\infty}\left(T^{*} M\right)$, and $\langle\xi\rangle^{-s} a$ extends to a smooth function on $\bar{T}^{*} M$. We then define the characteristic set of $A$ as $\left\{\langle\xi\rangle^{-s} a=0\right\} \subset \bar{T}^{*} M$ and say that $A$ is elliptic on some $U \subset \bar{T}^{*} M$ if $U$ does not intersect the characteristic set of $A$.

We use the definition of semiclassical wavefront set $\mathrm{WF}_{h}(A) \subset \bar{T}^{*} M$ for $A \in$ $\Psi^{S}(M)$ found in [28], Section 2, or [6], Section 2.1. The wavefront set of $A$ is empty if and only if $A$ lies in the algebra $h^{\infty} \Psi^{-\infty}(M)$ of smoothing operators such that each of $C^{\infty}(M \times M)$ seminorms of their Schwartz kernels decays faster than any power of $h$. For $A, B \in \Psi^{s}(M)$, we say that $A=B$ microlocally on some open $U \subset \bar{T}^{*} M$, if $\mathrm{WF}_{h}(A-B) \cap U=\emptyset$. Also, we say that $A \in \Psi^{s}(M)$ is compactly microlocalized, if $\mathrm{WF}_{h}(A)$ does not intersect the fiber infinity $\partial\left(\bar{T}^{*} M\right)$; in this case, $A \in \Psi^{s}(M)$ for all $s \in \mathbb{R}$.

We now recall several fundamental facts from semiclassical analysis.

Proposition 1 (Elliptic estimate). Let $P \in \Psi^{S}(M)$, be properly supported, $A \in$ $\Psi^{t}(M), t \leq s$, be compactly supported, and assume that $P$ is elliptic on $\mathrm{WF}_{h}(A) \subset$ $\bar{T}^{*} M$. Then there exists a compact set $K \subset M$ and a constant $C$ such that for each $u \in H_{\hbar, \mathrm{loc}}^{s}(M)$,

$$
\|A u\|_{L^{2}(M)} \leq C\|P u\|_{L^{2}(K)}+\mathcal{O}\left(h^{\infty}\right)\|u\|_{L^{2}(K)} .
$$

Proposition 2. Assume that $M$ is a manifold with prescribed volume form and $P(h) \in$ $\Psi^{s}$ is a properly supported self-adjoint operator on $L^{2}(M)$ with principal symbol $p \in C^{\infty}\left(T^{*} M ; \mathbb{R}\right)$. Let

$$
e^{i t P(h) / h}: L^{2}(M) \longrightarrow L^{2}(M), \quad t \in \mathbb{R},
$$

be the corresponding Schrödinger propagator, defined by means of spectral theory; it is a unitary operator. Let also $\exp \left(t H_{p}\right)$ be the Hamiltonian flow of $p$ on $T^{*} M$. Then (with constants below depending on $t$ ), we have the following results.

1 (Microlocalization). The operator $e^{i t P(h) / h}$ is microlocalized on the graph of $\exp \left(-t H_{p}\right)$ in the following sense: if $A, B \in \Psi^{0}(M)$ are compactly supported and $B$ is compactly microlocalized, and

$$
\exp \left(t H_{p}\right)\left(\mathrm{WF}_{h}(A)\right) \cap \mathrm{WF}_{h}(B)=\emptyset,
$$

then $A e^{i t P(h) / h} B=\mathcal{O}\left(h^{\infty}\right)_{L^{2} \rightarrow L^{2}}$.

2 (Egorov's Theorem). Let $A \in \Psi^{0}(M)$ be compactly supported and compactly microlocalized, with principal symbol a. Then there exists a compactly supported and compactly microlocalized operator $A_{t} \in \Psi^{0}(M)$ such that

$$
e^{i t P(h) / h} A e^{-i t P(h) / h}=A_{t}+\mathcal{O}\left(h^{\infty}\right)_{L^{2}(M) \rightarrow L^{2}(M)} .
$$


Moreover, $\mathrm{WF}_{h}\left(A_{t}\right) \subset \exp \left(-t H_{p}\right)\left(\mathrm{WF}_{h}(A)\right)$, and the principal symbol of $A_{t}$ is $a \circ \exp \left(t H_{p}\right)$.

A proof of Proposition 1 in the closely related microlocal case is given in Theorem 18.1.24' of [11]; see for example [6], Section 2.2, for the semiclassical case. For Proposition 2, see [34], Theorem 11.1, or [6], Proposition 2.6.

\section{Proofs}

We start by studying the equation (1.2) in some cusp $\ell_{j}$. Consider the Fourier series

$$
u \mid \boldsymbol{e}_{j}(r, \theta)=\sum_{k \in \mathbb{Z}} u_{k}^{j}(r) e^{i k \theta} .
$$

By (1.1), eq. (1.2) takes the form

$$
\left[\left(D_{r}+i / 2\right)^{2}+k^{2} e^{2 r}-\lambda^{2}\right] u_{k}^{j}(r)=0, \quad k \in \mathbb{Z} .
$$

For $k=0,(3.2)$ is a constant-coefficient ODE and we have

$$
u_{0}^{j}(r)=u_{+}^{j} e^{(1 / 2+i \lambda) r}+u_{-}^{j} e^{(1 / 2-i \lambda) r},
$$

for some constants $u_{ \pm}^{j}$.

Now, we extend the function $r$ smoothly from the union of all $\ell_{j}$ to the whole $M$ so that $r \leq R$ outside of the cusp regions. As before, let $1 e_{j}$ be the indicator function of the cusp $\ell_{j}$. Finally, fix a cutoff function $\tilde{\chi} \in C^{\infty}(\mathbb{R} ;[0,1])$ such that $\operatorname{supp} \tilde{\chi} \subset(0, \infty)$ and $\operatorname{supp}(1-\widetilde{\chi}) \subset(-\infty, 1)$.

Take a cusp $\ell_{j}$ and define the 'incoming' part of the Eisenstein function by

$$
E_{j}^{\prime}(\lambda)=1 e_{j} \cdot \tilde{\chi}(r-R) e^{(1 / 2-i \lambda) r} \in C^{\infty}(M) .
$$

Then

$$
F_{j}(\lambda)=\left(\Delta-1 / 4-\lambda^{2}\right) E_{j}^{\prime}(\lambda)=1 \varkappa_{j}[\Delta, \tilde{\chi}(r-R)] e^{(1 / 2-i \lambda) r} \in C_{0}^{\infty}(M) .
$$

Assume that for some constant $C_{0}$, we have

$$
C_{0}^{-1} \leq \operatorname{Im} \lambda \leq C_{0}, \quad \operatorname{Re} \lambda>1 .
$$

Since $\Delta$ is self-adjoint, the resolvent

$$
\left(\Delta-1 / 4-\lambda^{2}\right)^{-1}: L^{2}(M) \longrightarrow L^{2}(M)
$$

is well-defined and the only solution to (1.2) satisfying (1.3) is given by [18], Section 3,

$$
\begin{gathered}
E_{j}(\lambda)=E_{j}^{\prime}(\lambda)+E_{j}^{\prime \prime}(\lambda), \\
E_{j}^{\prime \prime}(\lambda)=-\left(\Delta-1 / 4-\lambda^{2}\right)^{-1} F_{j}(\lambda) .
\end{gathered}
$$

We can estimate $E_{j}^{\prime \prime}$ uniformly in $L^{2}(M)$. 
Proposition 3. There exists a constant $C$ such that for each $\lambda$ satisfying (3.5),

$$
\left\|E_{j}^{\prime \prime}(\lambda)\right\|_{L^{2}(M)} \leq C
$$

It follows that for each compact $K \subset M$, there exists a constant $C_{K}$ such that for each $\lambda$ satisfying (3.5),

$$
\left\|E_{j}(\lambda)\right\|_{L^{2}(K)} \leq C_{K}
$$

Proof. It follows from the definition of $F_{j}$ that $\left\|F_{j}\right\|_{L^{2}}=\mathcal{O}(|\lambda|)$. However, since $\Delta$ is self-adjoint,

$$
\left\|\left(\Delta-1 / 4-\lambda^{2}\right)^{-1}\right\|_{L^{2} \rightarrow L^{2}} \leq \frac{1}{\left|\operatorname{Im}\left(\lambda^{2}\right)\right|}=\mathcal{O}\left(|\lambda|^{-1}\right)
$$

and (3.7) follows. Next, (3.8) follows from (3.7) and the fact that $\left\|E_{j}^{\prime}\right\|_{L^{2}(K)} \leq$ $C_{K}$.

Also, $E_{j}$ is microlocalized on the cosphere bundle $S^{*} M$.

Proposition 4. Assume that $A(h) \in \Psi^{s}(M), s \leq 2$, is compactly supported and $\mathrm{WF}_{h}(A) \cap S^{*} M=\emptyset$. Let $h=(\operatorname{Re} \lambda)^{-1}$. Then $\left\|A(h) E_{j}(\lambda)\right\|_{L^{2}(M)}=\mathcal{O}\left(h^{\infty}\right)$.

Proof. Follows from Proposition 1 applied to (1.5) and (3.8).

We now prove the key technical estimate, approximating $E_{j}(\lambda)$ on a fixed compact set by the result of propagating the appropriately cut off 'incoming wave' $E_{j}^{\prime}(\lambda)$.

Proposition 5. Assume that $\lambda$ satisfies (3.5). As before, let $h=(\operatorname{Re} \lambda)^{-1}$. Then for $\operatorname{each} T>R, t>0$,

$$
\begin{aligned}
& \left\|\tilde{\chi}(T-r)\left(E_{j}(\lambda)-e^{i t h\left(1 / 4+\lambda^{2}\right)} e^{-i t h \Delta} \tilde{\chi}(T+2 t+1-r) E_{j}^{\prime}(\lambda)\right)\right\|_{L^{2}(M)} \\
& \quad=\mathcal{O}_{t, T}\left(h^{\infty}\right)+\mathcal{O}\left(e^{-2 t \operatorname{Im} \lambda}\right) .
\end{aligned}
$$

Here the $\mathcal{O}_{t, T}$ notation means that the constants in $\mathcal{O}(\cdot)$ depend on $t$ and $T$. The constant in $\mathcal{O}\left(e^{-2 t \operatorname{Im} \lambda}\right)$ is independent of $T, t, h$. Note also that $\tilde{\chi}(T-r) \in C_{0}^{\infty}(M)$.

Proof. First of all, we have by (3.7),

$$
\begin{gathered}
\left\|e^{i t h\left(1 / 4+\lambda^{2}\right)} e^{-i t h \Delta} \tilde{\chi}(T+2 t+1-r) E_{j}^{\prime \prime}(\lambda)\right\|_{L^{2}} \\
\leq e^{-2 t \operatorname{Im} \lambda}\left\|E_{j}^{\prime \prime}(\lambda)\right\|_{L^{2}}=\mathcal{O}\left(e^{-2 t \operatorname{Im} \lambda}\right) .
\end{gathered}
$$

Therefore, it suffices to prove that

$$
\left\|u_{t}\right\|_{L^{2}}=\mathcal{O}_{t, T}\left(h^{\infty}\right)
$$


where for $0 \leq s \leq t$, we define

$$
u_{s}=\tilde{\chi}(T-r)\left(E_{j}(\lambda)-e^{i s h\left(1 / 4+\lambda^{2}\right)} e^{-i s h \Delta} \tilde{\chi}(T+2 t+1-r) E_{j}(\lambda)\right) .
$$

Since $\tilde{\chi}(T-r) \tilde{\chi}(T+2 t+1-r)=\tilde{\chi}(T-r)$, we have $u_{0}=0$; next,

$$
\begin{aligned}
D_{s} u_{s} & =\tilde{\chi}(T-r) e^{i s h\left(1 / 4+\lambda^{2}\right)} e^{-i s h \Delta} h\left(\Delta-1 / 4-\lambda^{2}\right) \tilde{\chi}(T+2 t+1-r) E_{j}(\lambda) \\
& =\tilde{\chi}(T-r) e^{i s h\left(1 / 4+\lambda^{2}\right)} e^{-i s h \Delta} h[\Delta, \tilde{\chi}(T+2 t+1-r)] E_{j}(\lambda) .
\end{aligned}
$$

Let $X(h) \in \Psi^{0}$ be compactly supported and compactly microlocalized in a small neighborhood of the cosphere bundle $S^{*} M$, but equal to the identity microlocally near $\{r \leq T+2 t+1\} \cap S^{*} M$. Then by Proposition 4

$$
\left\|h[\Delta, \tilde{\chi}(T+2 t+1-r)](1-X(h)) E_{j}(\lambda)\right\|_{L^{2}}=\mathcal{O}_{t, T}\left(h^{\infty}\right) .
$$

Now, by Part 1 of Proposition 2,

$$
\left\|\widetilde{\chi}(T-r) e^{-i s h \Delta} h[\Delta, \tilde{\chi}(T+2 t+1-r)] X(h)\right\|_{L^{2} \rightarrow L^{2}}=\mathcal{O}_{t, T}\left(h^{\infty}\right) .
$$

To verify (2.1), we note that each point $\rho \in \mathrm{WF}_{h}(h[\Delta, \tilde{\chi}(T+2 t+1-r)] X(h))$ lies close to the cosphere bundle $S^{*} M$ (depending on the choice of $X(h)$ ) and inside $\{r>T+2 t\}$; therefore, by (1.6), the curve $\exp \left([0, t] H_{p}\right) \rho$ lies in $\mathscr{C}_{j} \cap\{r>T\}$, and thus does not intersect the support of $\tilde{\chi}(T-r)$. Here we use the fact that in each cusp region $\ell_{j}$, the derivative of the function $r$ along the geodesic flow is bounded by 1 , which can be verified directly using (1.1).

Since the operator in (3.11) is compactly supported, by (3.8) we get

$$
\left\|\tilde{\chi}(T-r) e^{-i s h \Delta} h[\Delta, \tilde{\chi}(T+2 t+1-r)] X(h) E_{j}(\lambda)\right\|_{L^{2}}=\mathcal{O}_{t, T}\left(h^{\infty}\right) .
$$

Combining (3.10) with (3.12), we arrive at

$$
\left\|d_{s} u_{s}\right\|_{L^{2}}=\mathcal{O}_{t, T}\left(h^{\infty}\right)
$$

integrating this from 0 to $t$, we get (3.9).

Armed with Proposition 5, we can make rigorous the 'proof' of the main theorem given in the introduction.

Proof of Theorem 1. Let $A \in \Psi^{0}$ be compactly supported with principal symbol $a$; it suffices to prove (1.8) and (1.9) without the cutoff $\chi$. We may assume that $A$ is compactly microlocalized; indeed, if $\mathrm{WF}_{h}(A) \cap S^{*} M=\emptyset$, then $\left\langle A E_{j}(\lambda), E_{j^{\prime}}(\lambda)\right\rangle=$ $\mathcal{O}\left(h^{\infty}\right)$ by Proposition 4 and (3.8). In fact, we may assume that $\mathrm{WF}_{h}(A)$ is contained in a small neighborhood of $S^{*} M$.

Fix $T>R$ such that $A$ is supported in $\{r<T-1\}$, so that

$$
A=\tilde{\chi}(T-r) A \tilde{\chi}(T-r) .
$$


By Proposition 5,

$$
\begin{gathered}
\tilde{\chi}(T-r) E_{j}(\lambda)=e^{i t h\left(1 / 4+\lambda^{2}\right)} \tilde{\chi}(T-r) e^{-i t h \Delta} \tilde{\chi}(T+2 t+1-r) E_{j}^{\prime}(\lambda) \\
+\mathcal{O}_{t}\left(h^{\infty}\right)_{L^{2}}+\mathcal{O}\left(e^{-2 t \operatorname{Im} \lambda}\right)_{L^{2}} .
\end{gathered}
$$

Take some $j, j^{\prime}$; substituting (3.13) into the expression

$$
\left\langle A E_{j}(\lambda), E_{j^{\prime}}(\lambda)\right\rangle=\left\langle A \tilde{\chi}(T-r) E_{j}(\lambda), \tilde{\chi}(T-r) E_{j^{\prime}}(\lambda)\right\rangle,
$$

and using the fact that the left-hand side of (3.13) is $\mathcal{O}(1)$ in $L^{2}$ by (3.8), we get

$$
\begin{gathered}
\left|\left\langle A E_{j}(\lambda), E_{j^{\prime}}(\lambda)\right\rangle-e^{-4 t \operatorname{Im} \lambda}\left\langle\widetilde{A_{t}} E_{j}^{\prime}(\lambda), E_{j^{\prime}}^{\prime}(\lambda)\right\rangle\right| \leq C e^{-v t}+\mathcal{O}_{t}\left(h^{\infty}\right), \\
\widetilde{A_{t}}=\tilde{\chi}(T+2 t+1-r) e^{i t h \Delta} A e^{-i t h \Delta} \tilde{\chi}(T+2 t+1-r),
\end{gathered}
$$

where we use that $\operatorname{Im} \lambda>v / 2$ for $h$ small enough. Here $C$ is a constant depending on $A$ and $T$, but not on $t$ or $h$. Therefore,

$$
\lim _{t \rightarrow+\infty} \limsup _{h \rightarrow 0}\left|\left\langle A E_{j}(\lambda), E_{j^{\prime}}(\lambda)\right\rangle-e^{-4 t \operatorname{Im} \lambda}\left\langle\widetilde{A_{t}} E_{j}^{\prime}(\lambda), E_{j^{\prime}}^{\prime}(\lambda)\right\rangle\right|=0,
$$

and thus

$$
\lim _{h \rightarrow 0}\left\langle A E_{j}(\lambda), E_{j^{\prime}}(\lambda)\right\rangle=\lim _{t \rightarrow+\infty} e^{-4 t \operatorname{Im} \lambda} \lim _{h \rightarrow 0}\left\langle\widetilde{A_{t}} E_{j}^{\prime}(\lambda), E_{j^{\prime}}^{\prime}(\lambda)\right\rangle,
$$

provided that the double limit on the right-hand side exists. To compute this limit, let $A_{t} \in \Psi^{0}$ be the compactly supported operator from part 2 of Proposition 2, with $P(h)=h^{2} \Delta$. Then $\mathrm{WF}_{h}\left(A_{t}\right) \subset\{r \leq T+2 t\}$; therefore,

$$
A_{t}=\tilde{\chi}(T+2 t+1-r) A_{t} \tilde{\chi}(T+2 t+1-r)+\mathcal{O}_{t}\left(h^{\infty}\right)_{L^{2} \rightarrow L^{2}}
$$

by (2.2), $\tilde{A}_{t}=A_{t}+\mathcal{O}_{t}\left(h^{\infty}\right)_{L^{2} \rightarrow L^{2}}$. Since both $A_{t}$ and $\tilde{A}_{t}$ are compactly supported, we can replace $\widetilde{A_{t}}$ by $A_{t}$ in (3.15). By (1.6), the principal symbol of $A_{t}$ on $S^{*} M$ is $a_{t}=a \circ \exp (2 t V)$.

For $j \neq j^{\prime}$, the supports of the functions $E_{j}^{\prime}(\lambda)$ and $E_{j^{\prime}}^{\prime}(\lambda)$ do not intersect (as they lie in different cusp regions); since $A_{t}$ is pseudodifferential and compactly supported, we get

$$
\left\langle A_{t} E_{j}^{\prime}(\lambda), E_{j^{\prime}}^{\prime}(\lambda)\right\rangle=\mathcal{O}_{t}\left(h^{\infty}\right)
$$

and (1.9) follows.

We now assume that $j=j^{\prime}$. We can use the definition (3.4) of $E_{j}^{\prime}$, the definition of semiclassical quantization, and the method of stationary phase to get for each $t$,

$$
\begin{gathered}
A_{t} E_{j}^{\prime}(\lambda)=1 e_{j}(a \circ \exp (2 t V))(r, \theta,-1,0) \tilde{\chi}(r-R) e^{(1 / 2+\operatorname{Im} \lambda) r} e^{-i r / h} \\
+\mathcal{O}_{t}(h)_{L_{\mathrm{comp}}^{2}} .
\end{gathered}
$$


Indeed, restricting to the cusp $\bigodot_{j}$, in local coordinates $(r, \theta)$ (we will also need to restrict to a topologically trivial subset of the circle, where $\theta \in \mathbb{R}$ gives a valid coordinate) the left-hand side of (3.16) becomes

$$
\begin{aligned}
& (2 \pi h)^{-2} \int e^{\frac{i}{h}\left(\left(r-r^{\prime}, \theta-\theta^{\prime}\right) \cdot\left(p_{r}, p_{\theta}\right)-r^{\prime}\right)} a_{t}\left(r, \theta ; p_{r}, p_{\theta}\right) \\
& \tilde{\chi}\left(r^{\prime}-R\right) e^{(1 / 2+\operatorname{Im} \lambda) r^{\prime}} d p_{r} d p_{\theta} d r^{\prime} d \theta^{\prime}+\mathcal{O}(h) .
\end{aligned}
$$

The stationary points of the phase $\Phi=\left(r-r^{\prime}, \theta-\theta^{\prime}\right) \cdot\left(p_{r}, p_{\theta}\right)-r^{\prime}$ are given by $r^{\prime}=r, \theta^{\prime}=\theta, p_{r}=-1, p_{\theta}=0$ and at these points, $\Phi$ takes the value $-r$ and its Hessian has determinant 1 and signature 0 . Applying the method of stationary phase, we get (3.16).

We now multiply (3.16) by $E_{j}^{\prime}(\lambda)$ and integrate, remembering that the volume form on $\mathcal{C}_{j}$ is given by $e^{-r} d r d \theta$ and $\operatorname{Im} \lambda \rightarrow v$ as $h \rightarrow 0$ :

$$
\begin{aligned}
\lim _{h \rightarrow 0} & \left\langle A_{t} E_{j}^{\prime}(\lambda), E_{j}^{\prime}(\lambda)\right\rangle \\
\quad & =\int_{\mathcal{C}_{j}} \tilde{\chi}(r-R)^{2} e^{2 v r}(a \circ \exp (2 t V))(r, \theta,-1,0) d r d \theta .
\end{aligned}
$$

Here $\left(r, \theta, p_{r}, p_{\theta}\right)$ are the coordinates on $T^{*} \mathcal{C}_{j}$ induced by the coordinate system $(r, \theta)$ on $\ell_{j}$. Letting $t \rightarrow+\infty$ and recalling (1.11), we get from (3.17)

$$
\lim _{h \rightarrow 0}\left\langle A E_{j}(\lambda), E_{j}(\lambda)\right\rangle=\int_{S^{*} M} a d \mu_{j v},
$$

which proves (1.8).

We can explain (3.16) using the theory of semiclassical Lagrangian distributions (see [10], Chapter 6, or [29], Section 2.3, for a detailed account, and [12], Section 25.1, or [8], Chapter 11, for the closely related microlocal case) as follows. By (3.4), the function

$$
E_{j}^{\prime}(\lambda)=1 e_{j} \cdot \tilde{\chi}(r-R) e^{(1 / 2+\operatorname{Im} \lambda) r} e^{-i r / h}
$$

is a Lagrangian distribution associated to the Lagrangian $\widehat{\mathcal{A}}_{j}^{-}$from (1.10), with the principal symbol $\tilde{\chi}(r-R) e^{(1 / 2+\operatorname{Im} \lambda) r}$. Since $A_{t}$ is pseudodifferential and compactly supported, $A_{t} E_{j}^{\prime}(\lambda)$ is also a Lagrangian distribution associated to $\hat{\mathcal{A}}_{j}^{-}$, and its principal symbol is the product of the principal symbol of $E_{j}^{\prime}(\lambda)$ and the restriction of the principal symbol of $A_{t}$ to $\hat{\mathcal{A}}_{j}^{-}$, proving (3.16).

Finally, we estimate the scattering coefficient by the mass of Eisenstein series on the outgoing set.

Proof of Theorem 2 . Fix a cusp $\ell_{j^{\prime}}$ and take compactly supported and compactly microlocalized $A \in \Psi^{0}((R, R+1))$ such that $\mathrm{WF}_{h}(A) \subset\left\{p_{r}>0\right\}$, and the principal symbol $a\left(r, p_{r}\right)$ of $A$ satisfies $a(R+1 / 2,1) \neq 0$. Let $\chi \in C_{0}^{\infty}(\mathbb{R})$ have $\chi(0)=1$. 
Denote $u(h)=E_{j}(\lambda(h))$ and recall that $S_{j j^{\prime}}(\lambda(h))=u_{+}^{j^{\prime}}(h)$ is defined by (3.3). We then have for each $\delta>0$,

$$
\left|u_{+}^{j^{\prime}}(h)\right|=\mathcal{O}(1)\left\|A u_{0}^{j^{\prime}}(h)\right\|_{L^{2}}+\mathcal{O}\left(h^{\infty}\right)=\mathcal{O}(1)\left\|A_{\delta} u(h)\right\|_{L^{2}(M)}+\mathcal{O}\left(h^{\infty}\right),
$$

uniformly in $\delta$, where $A_{\delta}=\chi\left(h D_{\theta} / \delta\right) A$ is a pseudodifferential operator supported in $\mathcal{C}_{j^{\prime}}$. However, by Theorem 1 we have, as $h \rightarrow 0$,

$$
\left\|A_{\delta} u(h)\right\|_{L^{2}(M)}^{2}=\left\langle A_{\delta}^{*} A_{\delta} u(h), u(h)\right\rangle_{L^{2}(M)} \rightarrow \int_{S^{*} \mathcal{C}_{j^{\prime}}}\left|\chi\left(h p_{\theta} / \delta\right) a\left(r, p_{r}\right)\right|^{2} d \mu_{j v} .
$$

By our assumption, $\mu_{j v}\left(\hat{\mathcal{A}}_{j^{\prime}}^{+}\right)=0$; therefore,

$$
\lim _{\delta \rightarrow 0} \lim _{h \rightarrow 0}\left\|A_{\delta} u(h)\right\|_{L^{2}(M)}=\int_{\hat{\mathcal{A}}_{j^{\prime}}^{+}}\left|a\left(r, p_{r}\right)\right|^{2} d \mu_{j v}=0
$$

and we are done by (3.18).

\section{Hyperbolic surfaces}

In this section, we consider the special case $M=\Gamma \backslash \mathbb{W}$, where $\mathbb{U}$ is the Poincaré half-plane model of the hyperbolic plane and $\Gamma \subset \operatorname{PSL}(2, \mathbb{R})$ is a Fuchsian group of the first kind, so that $M$ is a finite area hyperbolic surface. Denote by $\pi_{\Gamma}: \mathbb{U} \rightarrow M$ the projection map. The conformal boundary $\partial \mathbb{U}=\mathbb{R} \cup\{\infty\}$ is a circle, as can be seen by using the Poincaré ball model. This section is not used anywhere else in the paper and is provided as a quick reference for readers familiar with the theory of hyperbolic surfaces.

We first find an interpretation of (1.7) in terms of the group action; this is parallel to the representation of measures invariant under the Hamiltonian flow in PattersonSullivan theory (see for example [1], Section 14.2). We parametrize the cosphere bundle $S^{*}$ 叫 by

$$
\mathcal{T}:(\partial \mathbb{U} \times \partial \mathbb{U})_{\Delta} \times \mathbb{R} \rightarrow S^{*} \mathbb{U},
$$

where $(\partial \mathbb{U} \times \partial \mathbb{U})_{\Delta}$ is the Cartesian square of the circle $\partial \mathbb{U}$ minus the diagonal. The map $\mathcal{T}$ is defined as follows. Take $\left(q_{1}, q_{2}\right) \in(\partial \mathbb{U} \times \partial \mathbb{U})_{\Delta}$ and let $\gamma_{q_{1} q_{2}}(t)$ be the unique unit speed geodesic (that is, a semicircle in the half-plane model) going from $q_{1}$ to $q_{2}$, parametrized so that $\gamma(0)$ is the point of $\gamma$ closest to $i \in \mathbb{G}$. We put

$$
\mathcal{T}\left(q_{1}, q_{2}, t\right)=\left(\gamma_{q_{1} q_{2}}(t), \dot{\gamma}_{q_{1} q_{2}}(t)\right) .
$$

Now, consider a Radon measure $\mu$ on $S^{*} M$ satisfying (1.7). We can lift it to a measure $\mu^{\prime}$ on $S^{*} \mathbb{t}$; then

$$
\mathcal{T}^{*} \mu^{\prime}=\tilde{\mu} \times e^{-2 v t} d t
$$


where $\tilde{\mu}$ is some Radon measure on $(\partial \mathbb{U} \times \partial \mathbb{U})_{\Delta}$.

For each $\gamma \in \operatorname{PSL}(2, \mathbb{R})$, we can calculate

$$
\gamma\left(\mathcal{T}\left(q_{1}, q_{2}, t\right)\right)=\mathcal{T}\left(\gamma\left(q_{1}\right), \gamma\left(q_{2}\right), t+\frac{1}{2} \log \left|\frac{\gamma_{\mathbb{B}}^{\prime}\left(q_{1}\right)}{\gamma_{\mathbb{B}}^{\prime}\left(q_{2}\right)}\right|\right),
$$

where $\gamma_{\mathbb{B}}^{\prime}(q)$ is the derivative of $\gamma$ considered as a transformation on the ball model $\mathbb{B}$ with the identification map $\mathbb{U} \rightarrow \mathbb{B}$ given by $z \mapsto(z-i) /(z+i)$; if

$$
\gamma(z)=\frac{a z+b}{c z+d}, \quad\left(\begin{array}{ll}
a & b \\
c & d
\end{array}\right) \in \operatorname{SL}(2, \mathbb{R}),
$$

then

$$
\left|\gamma_{\mathbb{B}}^{\prime}(q)\right|=\frac{q^{2}+1}{(a q+b)^{2}+(c q+d)^{2}} .
$$

We see then that the measure $\mu^{\prime}$ defined by (4.1) is invariant under the action of $\Gamma$ on $S^{*} \mathbb{B}$ if and only if for each $\gamma \in \Gamma$,

$$
\gamma^{*} \tilde{\mu}=\left|\gamma_{\mathbb{B}}^{\prime}\left(q_{1}\right)\right|^{v}\left|\gamma_{\mathbb{B}}^{\prime}\left(q_{2}\right)\right|^{-v} \tilde{\mu},
$$

where

$$
\left(\gamma^{*} \tilde{\mu}\right)(A)=\tilde{\mu}((\gamma \times \gamma)(A)), A \subset(\partial \mathbb{B} \times \partial \mathbb{B})_{\Delta} .
$$

In particular, if $\hat{\mu}$ is a Radon measure on $\partial \mathbb{B}$ such that for each $\gamma \in \Gamma$,

$$
\gamma^{*} \hat{\mu}=\left|\gamma_{\mathbb{B}}^{\prime}(q)\right|^{2 v+1} \hat{\mu},
$$

then a measure $\tilde{\mu}$ satisfying (4.3) is given by (compare with eq. (14.14) in [1], bearing in mind that we use the half-plane model)

$$
\tilde{\mu}=\left|q_{1}-q_{2}\right|^{-2(v+1)}\left|q_{1}+i\right|^{2(v+1)}\left|q_{2}+i\right|^{2 v} \hat{\mu} \times d q_{2} .
$$

Now, fix a cusp region $\ell_{j}$ on $M$ and assume for simplicity that $\infty \in \partial \mathbb{\square}$ is a preimage of the corresponding cusp. Let $\Gamma_{\infty}$ be the group of all elements of $\Gamma$ fixing $\infty$; without loss of generality, we may assume that it is generated by the shift $z \rightarrow z+1$. Then all the preimages of the cusp of $\ell_{j}$ are given by

$$
\left\{\gamma(\infty) \mid \gamma \Gamma_{\infty} \in \Gamma / \Gamma_{\infty}\right\}
$$

i.e., they are indexed by right cosets of $\Gamma_{\infty}$ in $\Gamma$. Note that, for $\gamma$ given by (4.2),

$$
\gamma(\infty)=a / c,\left|\gamma_{\mathbb{B}}^{\prime}(\infty)\right|=\frac{1}{a^{2}+c^{2}} .
$$

A canonical system of coordinates on $\ell_{j}$ is given by

$$
(r, \theta) \in\left(R_{j}, \infty\right) \times \mathbb{S}^{1} \longmapsto \pi_{\Gamma}\left(\frac{\theta+i e^{r}}{2 \pi}\right) .
$$


Proposition 6. The lift of the measure $\mu_{j v}$ defined in (1.11) corresponds under (4.1) to $(2 \pi)^{2 v+1} \tilde{\mu}$, with $\tilde{\mu}$ given by (4.5), and

$$
\hat{\mu}=\sum_{\gamma \Gamma_{\infty} \in \Gamma / \Gamma_{\infty}} \frac{\delta_{a / c}}{\left(a^{2}+c^{2}\right)^{2 v+1}} ;
$$

here $\delta$ denotes a delta measure. (Note that the values $a / c$ are distinct for different cosets, as $\Gamma_{\infty}$ is the stabilizer of $\infty$.)

Proof. The measure $\hat{\mu}$ is well-defined, as the series

$$
\sum_{\gamma \Gamma_{\infty} \in \Gamma / \Gamma_{\infty}} \frac{1}{\left(a^{2}+c^{2}\right)^{2 v+1}}=\sum_{\gamma \Gamma_{\infty} \in \Gamma / \Gamma_{\infty}}\left(\operatorname{Im} \gamma^{-1}(i)\right)^{2 v+1}
$$

converges, by convergence of Eisenstein series (4.10). By (4.6), the measure $\hat{\mu}$ satisfies (4.4); therefore, it produces a measure $\mu$ supported on the cosphere bundle $S^{*} M$ and satisfying (1.7). Moreover, since $\hat{\mu}$ is supported on the set of the preimages of the cusp of $\mathcal{C}_{j}, \mu$ is supported on $\mathcal{A}_{j}^{-}$. It then suffices to study the restriction of $\mu$ to $\widehat{\mathcal{A}}_{j}^{-}$. To this end, take $A \subset\left(R_{j}, \infty\right) \times \mathbb{S}^{1}$ and consider

$$
\widetilde{A}=\left\{(r, \theta ;-1,0) \in T^{*} \boldsymbol{C}_{j} \mid(r, \theta) \in A\right\} \subset \widehat{\mathcal{A}}_{j}^{-} .
$$

Since

we get

$$
\mathcal{T}(\infty, q, t)=\left(q+i|i+q| e^{-t},-i \frac{e^{t}}{|i+q|}\right)
$$

$$
\begin{gathered}
\tilde{A}=\pi_{\Gamma} \mathcal{T}(\{(\infty, q, t) \mid(q, t) \in \breve{A}\}), \\
\check{A}=\{(\theta /(2 \pi),-r+\ln (2 \pi)+\ln |i+\theta /(2 \pi)|) \mid(r, \theta) \in A\} .
\end{gathered}
$$

Then

$$
\mu(\widetilde{A})=\int_{(q, t) \in \check{A}}|i+q|^{2 v} e^{-2 v t} d q d t=(2 \pi)^{-2 v-1} \int_{A} e^{2 v r} d r d \theta
$$

and the proof is finished by the definition (1.11) of $\mu_{j v}$.

In particular, for the modular surface the measure $\hat{\mu}$ is given by

$$
\hat{\mu}=\sum_{\substack{m, n \in \mathbb{Z} \\ n \geq 0, m \perp n}} \frac{\delta_{m / n}}{\left(m^{2}+n^{2}\right)^{2 v+1}} .
$$

Finally, we note that for $v>1 / 2$ one can prove Theorem 1 for hyperbolic surfaces using the series representation for the Eisenstein function

$$
\widetilde{E}(\lambda ; z)=(2 \pi)^{1 / 2-i \lambda} \sum_{\Gamma_{\infty} \gamma \in \Gamma_{\infty} \backslash \Gamma}(\operatorname{Im} \gamma(z))^{1 / 2-i \lambda}, \quad z \in \mathbb{H} .
$$


This series converges absolutely (see [15], Theorem 2.1.1); since it is invariant under $\Gamma$ and each of its terms solves (1.2) on $\mathbb{H}$, it gives rise to a solution $\widehat{E}(\lambda, z)$ of (1.2). It can also be seen that (4.10) converges in $L^{2}$ of a fundamental domain of $\Gamma$, if we take out the term with $\gamma=1$; therefore, $\widehat{E}(\lambda, z)$ satisfies (1.3) and we have

$$
\widetilde{E}(\lambda ; z)=E_{j}(\lambda ; \pi(z)) .
$$

The $(2 \pi)^{1 / 2-i \lambda}$ prefactor here is due to the fact that in our normalization of the Eisenstein series, the incoming term is given by $e^{(1 / 2-i \lambda) r}$, and a canonical system of coordinates is given by (4.7), with a $2 \pi$ factor there since the stabilizer of the cusp is generated by the transformation $z \mapsto z+1$, while we need the $\{r=0\}$ circle to have length $2 \pi$.

One then proceeds as in the proof of Theorem 2 of [9], by analysing the microlocal limit of each term of the Eisenstein series and showing that the off-diagonal terms of the sum $\langle A \widetilde{E}, \widetilde{E}\rangle$ are negligible. (The analysis of [9] is dramatically simplified, as we are not asking for an estimate on the remainder and thus one can sum over an $h$-independent number of the elements of the group in Lemma 7 of [9] and use standard microlocal analysis.)

\section{References}

[1] D. Borthwick, Spectral theory of infinite-area hyperbolic surfaces. Birkhäuser, Basel, 2007. MR 2344504 Zbl 1130.58001

[2] Y. Colin de Verdière, Pseudo-Laplaciens II. Ann. Inst. Fourier 33 (1983), 87-113. MR 699488 Zbl 0496.58016 www.numdam.org/item?id=AIF_1983_33_2_87_0

[3] Y. Colin de Verdière, Ergodicité et fonctions propres du Laplacien. Commun. Math. Phys. 102 (1985), 497-502. MR 818831 Zbl 0592.58050

projecteuclid.org/getRecord?id=euclid.cmp/1104114465

[4] K. Datchev and A. Vasy, Propagation through trapped sets and semiclassical resolvent estimates. To appear in Ann. Inst. Fourier. Preprint 2010. arXiv:1010.2190

[5] M.F. Demers and L.-S. Young, Escape rates and conditionally invariant measures. Nonlinearity 19 (2006), 377-397. MR 2199394 Zbl 1134.37322

[6] S. Dyatlov, Asymptotic distribution of quasi-normal modes for Kerr-de Sitter black holes. To appear in Ann. Inst Henri Poincaré Preprint 2011. arXiv:1101.1260

[7] P. Gérard and É. Leichtnam, Ergodic properties of eigenfunctions for the Dirichlet problem. Duke Math. J. 71 (1993), 559-607. MR 1233448 Zbl 0788.35103

[8] A. Grigis and J. Sjöstrand, Microlocal analysis for differential operators. An introduction. Cambridge University Press, Cambridge, 1994. MR 1269107 Zbl 0804.35001

[9] C. Guillarmou and F. Naud, Equidistribution of Eisenstein series on convex co-compact hyperbolic manifolds. Preprint 2011. arXiv:1107.2655

[10] V. Guillemin and S. Sternberg, Geometric asymptotics. Amer. Math. Soc., Providence (RI), 1977. MR 0516965 Zbl 0364.53011 
[11] L. Hörmander, The Analysis of linear partial differential operators III. Pseudo-differential Operators. Springer Verlag, Berlin etc., 1985. MR 2304165 Zbl 0601.35001

[12] L. Hörmander, The analysis of linear partial differential operators IV. Fourier Integral Operators. Springer Verlag, Berlin etc., 1985. MR 781537 Zbl 0612.35001

[13] D. Jakobson, Quantum unique ergodicity for Eisenstein series on $\operatorname{PSL}_{2}(\mathbb{Z}) \backslash \mathrm{PSL}_{2}(\mathbb{R})$. Ann. Inst. Fourier 44 (1994), 1477-1504. MR 1313792 Zbl 0820.11040 www.numdam.org/item?id=AIF_1994_44_5_1477_0

[14] J. P. Keating, S. Nonnenmacher, M. Novaes, and M. Sieber, On the resonance eigenstates of an open quantum baker map. Nonlinearity 21 (2008), 2591-2624. MR 2448233 Zbl 1158.35319

[15] T. Kubota, Elementary theory of Eisenstein series. John Wiley \& Sons, New York etc., 1973. MR 0429749 Zbl 0268.10012

[16] E. Lindenstrauss, Invariant measures and arithmetic quantum unique ergodicity. Ann. Math. (2) 163 (2006), 165-219. MR 2195133 Zbl 1104.22015

[17] W. Luo and P. Sarnak, Quantum ergodicity of eigenfunctions on $\mathrm{PSL}_{2}(\mathbb{Z}) \backslash \mathbb{\boxplus}^{2}$. Publ. Math., Inst. Hautes Étud. Sci. 81 (1995), 207-237. MR 1361757 Zbl 0852.11024 www.numdam.org/item?id=PMIHES_1995_81_207_0

[18] W. Müller, Spectral geometry and scattering theory for certain complete surfaces of finite volume. Invent. Math. 109 (1992), 265-305. MR 1172692 Zbl 0772.58063

[19] S. Nonnenmacher, Spectral problems in open quantum chaos. Nonlinearity 24 (2011), 123-167. iopscience.iop.org/0951-7715/24/12/R02/

[20] S. Nonnenmacher and M. Rubin, Resonant eigenstates in quantum chaotic scattering. Nonlinearity 20 (2007), 1387-1420. MR 2327130 Zbl 1138.81021

[21] S. Nonnenmacher and M. Zworski, Quantum decay rates in chaotic scattering. Acta Math. 203 (2009), 149-233. MR 2570070 Zbl 1226.35061 www.springerlink.com/content/dm3g74643372413j/

[22] Y. N. Petridis, N. Raulf, and M. S. Risager, Quantum limits of Eisenstein series and scattering states. Preprint 2011. arXiv:1111.6615

[23] R. S. Phillips and P. Sarnak, On cusp forms for co-finite subgroups of PSL(2, R). Invent. Math. 80 (1985), 339-364. MR 788414 Zbl 0558.10017

[24] P. Sarnak, Recent progress on the quantum unique ergodicity conjecture. Bull. Amer. Math. Soc., New. Ser. 48 (2011), 211-228. MR 2774090 Zbl 05879836 www.ams.org/journals/bull/2011-48-02/S0273-0979-2011-01323-4/

[25] A. I. Schnirelman, Ergodic properties of eigenfunctions. Usp. Mat. Nauk. 29 (1974), 181-182. MR 0402834 Zbl 0324.58020

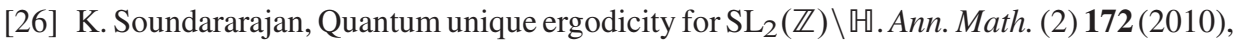
1529-1538. MR 2680500 Zbl 1209.58019

[27] E.C. Titchmarsh, The theory of the Riemann zeta-function. second ed., Clarendon Press, Oxford, 1986. MR $882550 \mathrm{Zbl} 0601.10026$

[28] A. Vasy, Microlocal analysis of asymptotically hyperbolic spaces and high energy resolvent estimates. Prerpint 2011. arXiv:1104.1376 
[29] S. Vũ Ngọc, Systèmes intégrables semi-classiques: du local au global. Société Mathématique de France, Paris, 2006. MR 2331010 Zbl 1118.37001

[30] S. Zelditch, Uniform distribution of eigenfunctions on compact hyperbolic surfaces. Duke Math. J. 55 (1987), 919-941. MR 916129 Zbl 0643.58029

[31] S. Zelditch, Mean Lindelöf hypothesis and equidistribution of cusp forms and Eisenstein series. J. Funct. Anal. 97 (1991), 1-49. MR 1105653 Zbl 0743.58034

[32] S. Zelditch, Recent developments in mathematical quantum chaos. In D. Jerison et al. (eds.), Current developments in mathematics, 2009. International Press, Somerville (MA), 2010, 115-204. MR 2757360 Zbl 1223.37113

[33] S. Zelditch and M. Zworski, Ergodicity of eigenfunctions for ergodic billiards. Commun. Math. Phys. 175 (1996), 673-682. MR 1372814 Zbl 0840.58048

projecteuclid.org/getRecord?id=euclid.cmp/1104276097

[34] M. Zworski, Semiclassical analysis. to be published by Amer. Math. Soc., Providence (RI). Preprint math.berkeley.edu/ zworski/semiclassical.pdf

Received December 6, 2011; revised December 28, 2011

Semyon Dyatlov, Department of Mathematics, Evans Hall, University of California,

Berkeley, CA 94720, U.S.A.

E-mail: dyatlov@math.berkeley.edu 